\title{
Modelo de difusión de cloruros en las estructuras de hormigón armado expuestas en la Península de Yucatán (México) ${ }^{(\bullet)}$
}

\author{
H. Castañeda ${ }^{(*)}$, P. Castro ${ }^{(* *)}$, C. González ${ }^{(*)}$ y J. Genescá ${ }^{(*)}$ \\ Resumen La corrosión del acero de refuerzo es una de las causas que reduce la vida en servicio de las estructu- \\ ras de hormigón, especialmente en las zonas costeras. Este fenómeno, en atmósferas marinas, se pro- \\ duce como consecuencia de la llegada de iones cloruro a la superficie de la barra por difusión o capi- \\ laridad. La vida útil de una estructura se puede dividir en dos períodos: uno de iniciación, durante el \\ cual el acero se mantiene pasivo como resultado del contacto con un medio de $\mathrm{pH}$ alcalino, en el cual \\ los iones agresivos penetran el hormigón en dirección al acero, y otro período de propagación, que se \\ inicia cuando la concentración de los iones cloruro en la superficie del acero excede de un cierto \\ nivel crítico. El propósito de este trabajo es presentar un modelo, basado en la segunda ley de Fick, \\ que permita estudiar la difusión de los iones cloruro en un medio marino durante el período de inicia- \\ ción. Se toma en consideración tanto la variabilidad de los coeficientes de difusión como los paráme- \\ tros inherentes al hormigón, durante un tiempo de exposición de 2 años en las condiciones ambienta- \\ les prevalecientes en la Península de Yucatán (México). Para la resolución del conjunto de \\ ecuaciones a que da lugar el modelo se ha utilizado el método numérico de las diferencias finitas, \\ teniéndose en cuenta la no linealidad del problema. Los valores obtenidos a partir del modelo se han \\ comparado con los experimentales para así comprobar la efectividad y viabilidad del modelo pro- \\ puesto.
}

Palabras clave: Coeficiente de difusión de cloruros. Atmósfera marina. Modelo matemático. Método de diferencias finitas. Hormigón armado.

\section{Mathematical model for chloride diffusion in reinforced concrete structures at Yucatán Peninsula, México}

\begin{abstract}
Corrosion of reinforcing steel can seriously limit the service life of concrete structures in marine applications. Chloride diffusion is one of the two major problems causing this phenomenon, increasing when the concrete is exposed to a marine environment in coastal areas. The service life of a structure may be divided into two general phases: an initiation period, in which the reinforcing steel surface is still passive as a result of the contact with a high $\mathrm{pH}$ medium, in which the aggressive ions penetrate the concrete toward the reinforcing bar, and a propagation period that starts when the chloride ion contamination on the steel surface exceeds the critical threshold level for the passivity breakdown. The aim of this work is to examine the chloride diffusion at the initial period on a marine environment by considering the variability of the diffusion coefficients with inherent parameters of the concrete, exposed during a period of two years in Yucatán, México. A model based on the second Fick's law is proposed; the non-linearity of the problem is taken into account and a numerical procedure based on the finite difference method is developed to solve the set of equations. Comparisons with experimental data are also carried out in order to show the reliability and the effectiveness of the proposed numerical model.
\end{abstract}

Keywords: Chloride diffusion coefficient. Marine environment. Mathematical model. Corrosion. Durability. Finite differences method. Reinforced concrete.

(•) Trabajo recibido el día 10 de febrero de 1997.

*) Dpto. de Ingeniería Metalúrgica. Facultad de Química. Universidad Nacional Autónoma de México (UNAM). 04510 México D.F. (México).

(**) CINVESTAV. Unidad Mérida, km 6. Ant. Carretera a Progreso, A.P., 73, Cordemex, C.P. 97310 Mérida, Yuc. (México).

\section{INTRODUCCIÓN}

México es uno de los muchos países que sufre problemas de corrosión en sus infraestructuras (1), con mayor severidad en las costas o ambientes marinos. Dichos problemas no se han evaluado, 
hecho que provoca pérdidas tanto humanas como económicas. Los tipos de corrosión se manifiestan de diferentes formas, pero, en ambientes marinos, el principal agente que acelera este fenómeno es el ion cloruro proveniente del mar. En el caso de las estructuras de hormigón armado, el daño es poco visible a edades tempranas y la aparición de herrumbre es el primer signo visible de corrosión avanzada. Además, en la Península de Yucatán, en la preparación del hormigón se utilizan materiales calcáreos y relaciones agua/cemento $(\mathrm{a} / \mathrm{c})$ elevadas, que tienen acusada influencia en el desarrollo de la corrosión de la armadura. De aquí, la importancia de contar con herramientas que permitan predecir vidas útiles residuales de las estructuras.

La predicción cuantitativa de la vida útil de una estructura es un paso crucial en el análisis costobeneficio necesario para determinar la durabilidad de la misma. Cualquier predicción se refiere, por lo regular, a la velocidad de acumulación del cloruro en la superficie de las armaduras embebidas en hormigón y al cálculo, mediante un programa de ordenador, del tiempo necesario para llegar a la concentración crítica (momento del rompimiento de la capa pasiva formada) en la interfase acero-hormigón. Para el presente trabajo se consideró como la concentración crítica de cloruros necesaria para el inicio de la corrosión y, de acuerdo a la experiencia previa de los autores, el intervalo comprendido entre 0,6 y $0,9 \mathrm{~kg} / \mathrm{m}^{3}$, que concuerda con valores similares publicados recientemente (2).

Una de las herramientas utilizadas actualmente por la ingeniería para realizar predicciones e interpretar comportamientos de materiales es el modelado matemático, cuyos resultados, especialmente cuando se comparan y validan con los experimentales obtenidos directamente en el campo, son de gran utilidad y ayuda para el estudio y seguimiento de los procesos de corrosión en las estructuras de hormigón armado.

Una relación de algunos parámetros no contemplados por algunos de los modelos ya publicados en la literatura (3-5), es la siguiente: la variación de la concentración de iones cloruro en la superficie con respecto al tiempo, la heterogeneidad del sistema debida a los agregados del hormigón, el transporte no difusional del agua en los poros del hormigón y los iones cloruro, la presencia de grietas en el hormigón, la presencia de esfuerzos, el efecto del gradiente de las porosidades, la temperatura, el potencial eléctrico, los cambios locales en la concentración crítica debidos a la polarización del acero en la celda de corrosión formada, el efecto de formas geométricas complicadas (en el caso de no ser estas simples), y el efecto del atrapamiento del ion cloruro por mecanismos tales como el entrelazamiento químico.

En este trabajo se parte de una forma geométrica simple, como es el caso de un cilindro. Sin embargo, se ha tomado en consideración en el modelo la complejidad del fenómeno al introducir un ciclo de mojado y secado en la superficie del hormigón, durante el cual se tendrá una concentración variable en ésta debido a los cambios climáticos. Se asume que el transporte del ion cloruro a través del hormigón tiene lugar por un proceso de difusividad, dependiente del tiempo y de otros parámetros, tales como la relación a/c y el tiempo de curado. La aplicación de soluciones estándar de la ecuación de Fick (segunda ley) en una dimensión, ha permitido realizar la predicción del tiempo necesario para alcanzar una determinada concentración de cloruros en la interfase armadura-hormigón durante el período de iniciación del proceso de corrosión. Esta aproximación es una propuesta útil para estimar el perfil de penetración de cloruros en el hormigón armado. Puede ser de gran ayuda para predecir el inicio de la etapa de propagación, aunque se tiene que reconocer que la no inclusión de otros parámetros relacionados con el comportamiento de una estructura real de hormigón armado afecta a la precisión de las predicciones.

\section{HIPÓTESIS BÁSICAS Y DESCRIPCIÓN DEL MODELO}

En el modelo matemático para la simulación del sistema acero-hormigón se han adoptado algunas suposiciones significativas con el fin de obtener resultados reproducibles. Se ha considerado la difusión de los iones cloruro dentro de la matriz de hormigón y se ha tomado en cuenta el efecto que tiene la variación del contenido de $\mathrm{Cl}^{-}$en el medio ambiente como resultado de las condiciones climáticas predominantes en cada estación del año sobre el perfil de concentraciones encontrado. Para validar el modelo, se han determinado experimentalmente los perfiles de concentración de cloruros libres que difunden en estructuras de hormigón simple (sin armaduras) en las costas de Progreso (Yucatán) a través del tiempo.

En el modelo propuesto se han considerado las siguientes restricciones y suposiciones:

- Se supone que en la degradación del hormigón armado influyen solamente los cloruros libres, estando estos disueltos en el agua del poro.

- Se considera un elemento de forma geométrica simple (cilíndrica), sin formar este un par galvánico con alguna parte de otra estructura de hormigón. Se asume que este elemento tiene una distribución de poro homogénea a lo largo del mismo. Los efectos de agrietamiento se ignoraron a lo largo del elemento de interés.

- Tanto la actividad del ion cloruro como la temperatura se mantienen constantes con el 
tiempo. Asimismo, se consideran despreciables los efectos de la carbonatación.

- Se asume que el proceso de difusión de los iones cloruro en un elemento cilíndrico poroso de hormigón es unidireccional y en forma de curvas concéntricas a partir de la superficie hacia el centro, obedeciendo a la segunda ley de Fick en una dimensión para coordenadas cilíndricas:

$$
\frac{\partial C}{\partial t}=D(t)\left(\frac{\partial^{2} C}{\partial r^{2}}=\frac{1 \partial C}{r \partial r}\right)
$$

bajo las siguientes condiciones de frontera:

$$
\begin{array}{ll}
t=0 ; & C=0 \\
r=0 ; & \frac{\partial C}{\partial r}=0 \\
r=r_{0} ; & C=C_{s}(t)
\end{array}
$$

El coeficiente de difusión, representado por $D(t)$, es dependiente del tiempo y de otras variables como la relación agua-cemento $(\mathrm{a} / \mathrm{c})$ y de los días de curado (d.c.). La concentración en la superficie es una condición de frontera del sistema y se representa como un término $C_{\mathrm{s}}(t)$, no lineal, que depende del tiempo.

\subsection{Modelo de difusión de los iones cloruro}

\subsubsection{Coeficiente de difusión de los iones cloruro en hormigón}

Una característica que distingue la difusión de los cloruros en el hormigón de un proceso de difusión de cloruros ordinario es la dependencia del coeficiente de difusión con el tiempo. El comportamiento del coeficiente de difusión se puede expresar en los términos de una ecuación matemática que obedezca el comportamiento experimental del sistema y que proponga una relación que dependa de la variable independiente elegida, como es el caso del tiempo, $D(t)$, expresado en $\mathrm{cm}^{2} / \mathrm{s}$.

\subsubsection{Concentración en la superficie}

En el hormigón fresco, la concentración de cloruros en la superficie es, por lo regular, muy pequeña. Cuando el hormigón está expuesto a ambientes marinos, la concentración en su superficie empieza a aumentar. Sin embargo, la condición a la frontera utilizada en este trabajo para la superficie del hormigón simula la exposición del mismo a atmósferas marinas, considerando así el efecto de las condiciones climáticas. En estos ambientes, los cloruros son transportados por medio del aire a la superficie en forma de pequeñas gotas de agua de mar. La concentración del ion en la capa superficial es controlada tanto por el proceso de depositación de los cloruros presentes en el aire como por el efecto contrario de lavado por el agua de la lluvia (6). La cantidad de iones depositados o lavados en una unidad de tiempo y por unidad de área se puede representar por medio de expresiones no lineales que permiten ajustar el comportamiento experimental mostrado por las estructuras expuestas en la atmósfera marina estudiada, obteniéndose una expresión en la que la concentración superficial es dependiente del tiempo y de los parámetros utilizados en la elaboración del hormigón mismo, como la relación a/c y los días de curado.

\subsection{Formulación matemática}

La solución de la ec. [1] aplicada al sistema hormigón armado se obtuvo numéricamente, empleando una aproximación convencional del método de diferencias finitas. Se tomó en cuenta un coeficiente de difusión $D(t)$ dependiente del tiempo, así como una dependencia de la concentración de cloruros con respecto a las condiciones climáticas, reflejadas en la concentración superficial de estos. El algoritmo desarrollado permite que, tanto la expresión no lineal del coeficiente de difusión como la condición de frontera propuesta para la superficie, puedan ser consideradas en la generación del perfil de penetración de los iones cloruro como una función del tiempo. Mediante un programa de cómputo, elaborado en QBasic, se implementó este algoritmo con el fin de realizar una simulación que permitiera reproducir los resultados encontrados experimentalmente.

\section{PROCEDIMIENTO EXPERIMENTAL}

Para este trabajo se utilizaron probetas cilíndricas de hormigón simple (sin varilla de refuerzo). Se diseñaron cinco mezclas correspondientes a las relaciones a/c: $0,76,0,70,0,53,0,50$ y 0,46 , y 3 tiempos de curado, 1, 3 y 7 días (7). Sus dimensiones eran de $8,0 \mathrm{~cm}$ de diámetro $\times 15 \mathrm{~cm}$ de altura.

Se utilizaron los tiempos de curado de 1,3 y 7 días por ser los normales en la zona del estudio, la Península y el Estado de Yucatán. En esta, para las casas-habitación y construcciones sin supervisión, se utilizan tiempos de curado de un día y, en algunos pocos casos, de 3 días. Para construcciones 
como escuelas y edificios grandes, se especifica un tiempo de 7 a 28 días de curado, pero cuando mucho, se llega a 7 días. Se determinó el contenido de cloruros en las probetas antes de exponerlas al medio ambiente marino y, posteriormente, a los 6 , 12 y 24 meses de exposición. Los cilindros se recubrieron con una capa gruesa de pintura de alquitrán de hulla en cada una de sus bases para que la penetración de cloruros tuviera lugar predominantemente en el sentido radial. Para tomar muestras se procedió a cortar una sección de $5 \mathrm{~mm}$ de espesor del cilindro, obteniéndose los polvos correspondientes a siete profundidades diferentes de cada muestra, y se les extrajeron los cloruros en medio ácido, empleándose un electrodo selectivo de cloruros para determinar la concentración.

\section{RESULTADOS EXPERIMENTALES Y DISCUSIÓN}

\subsection{El coeficiente de difusión de cloruros en hormigón}

Existe un gran número de factores que afectan al coeficiente de difusión de cloruros en un hormigón simple. La porosidad del hormigón es uno de los más importantes. Después de su amasado, el proceso de curado (o de hidratación) comienza inmediatamente. Durante este período de curado se forman los poros, que no tienen ni un tamaño ni una distribución definida. La figura 1 muestra el comportamiento del coeficiente de difusión con respecto al tiempo. La curva A representa el coeficiente de difusión en hormigón tipo Portland (OPC) con una relación agua-cemento (a/c) de 0,46 para 7 días de curado (d.c.); la curva B muestra el comportamiento para un OPC con una relación a/c de 0,70 y 7 d.c; finalmente, la curva $C$ muestra el comportamiento para un OPC con una relación a/c de 0,76 y 1 d.c. Se encontró que las curvas se pueden representar con las siguientes expresiones no lineales para cada una:

Curva A: $\quad D(t)=\operatorname{Exp}(-13,1094243-0,14222648 \times t)$

Curva B: $\quad D(t)=\operatorname{Exp}(-12,779273-0,45143268 \times t)$

Curva C: $D(t)=\operatorname{Exp}(-12,7502069-0,21645562 \times t)$

donde $D(t)$ es el coeficiente de difusión, en $\mathrm{cm}^{2} / \mathrm{s}$, y $t$ el tiempo, en meses. Cada ecuación corresponde a la representación matemática de la curva, ajustada a partir de los datos experimentales correspondientes a un período de dos años.

En la figura 2 se muestra el comportamiento del coeficiente de difusión con respecto al tiempo de curado para un OPC con una relación a/c de 0,46

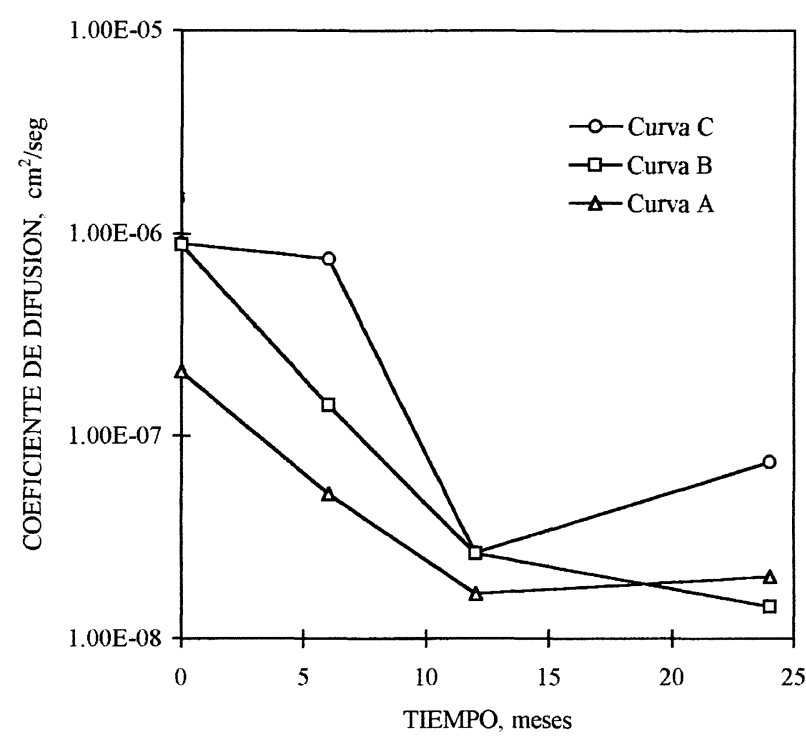

FIG. 1.- Coeficiente de difusión de los iones cloruro en un cilindro de hormigón (datos experimentales) en función del tiempo de exposición. Curva A: relación a/c: 0,46, 7 d.c. Curva $B$ : relación a/c: 0,70, 7 d.c. Curva C: relación a/c: 0,76, 1 d.c.

FIG. 1.-Diffusion coefficient of chloride ions on a concrete cylinder (experimental data) as a function of exposure time. Curve A: w/c: 0.46, curing time: 7 days. Curve B: $w / c: 0.70$, curing time: 7 days. Curve $C: w / c: 0.76$, curing time: 1 day.

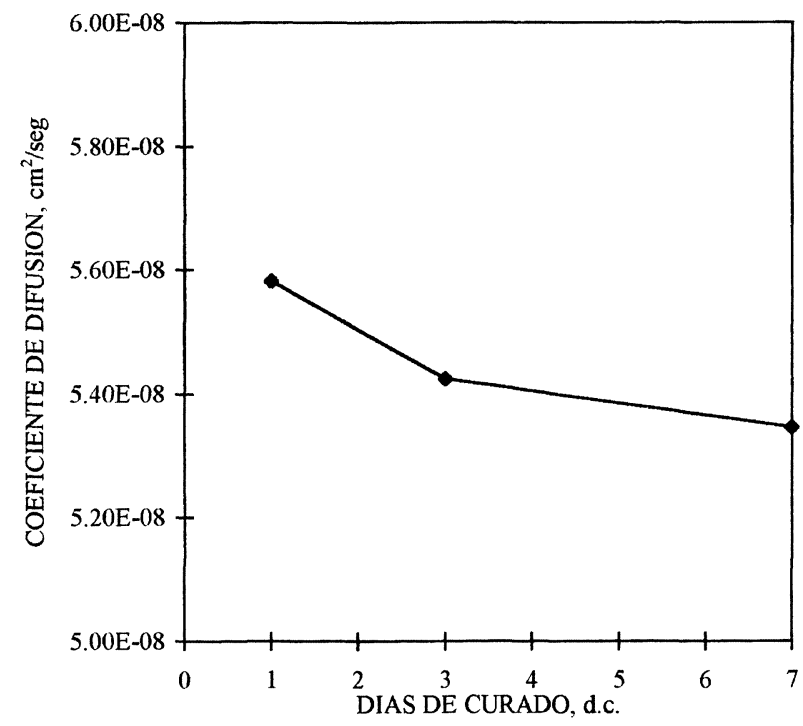

FIG. 2.- Coeficiente de difusión de los iones cloruro en función del tiempo de curado del hormigón; relación a/c 0,46 y tiempo de exposición 6 meses.

FIG. 2.-Diffusion coefficient of chloride ions as a function of concrete curing time; w/c: 0.46, exposure time: 6 months.

durante un tiempo de exposición de 6 meses, el cual se puede representar con la siguiente expresión: 
$D($ d.c. $)=(6,8501493+(1,5577612 / d c)) \times 1 \mathrm{E}-08$

donde $D$ es el coeficiente de difusión (en $\mathrm{cm}^{2} / \mathrm{s}$ ) y (d.c.) el tiempo de curado (en días). Esta ecuación es la representación de la curva ajustada a partir de los datos experimentales en un período de 2 años para las probetas de hormigón simple.

\subsection{Concentración en la superficie}

La figura 3 muestra el comportamiento de la concentración de los iones cloruro en la superficie del hormigón con respecto al tiempo. La curva A representa la concentración superficial para un OPC con una relación a/c de 0,46 y 7 d.c.; la curva $B$ muestra el comportamiento para un OPC con una relación a/c de 0,7 y 7 d.c.; finalmente, la curva $\mathrm{C}$ muestra el comportamiento para un OPC con una relación a/c de 0,76 y 1 d.c. Las curvas se pueden representar mediante la siguiente expresión:

$$
C_{\mathrm{s}}(t)=C_{\text {mín. }}+\left(C_{\text {máx. }}-C_{\text {mín }}\right) \times \operatorname{sen}(\theta)
$$

$\operatorname{con} \theta=(\Pi / 6) \times t^{\prime}$,

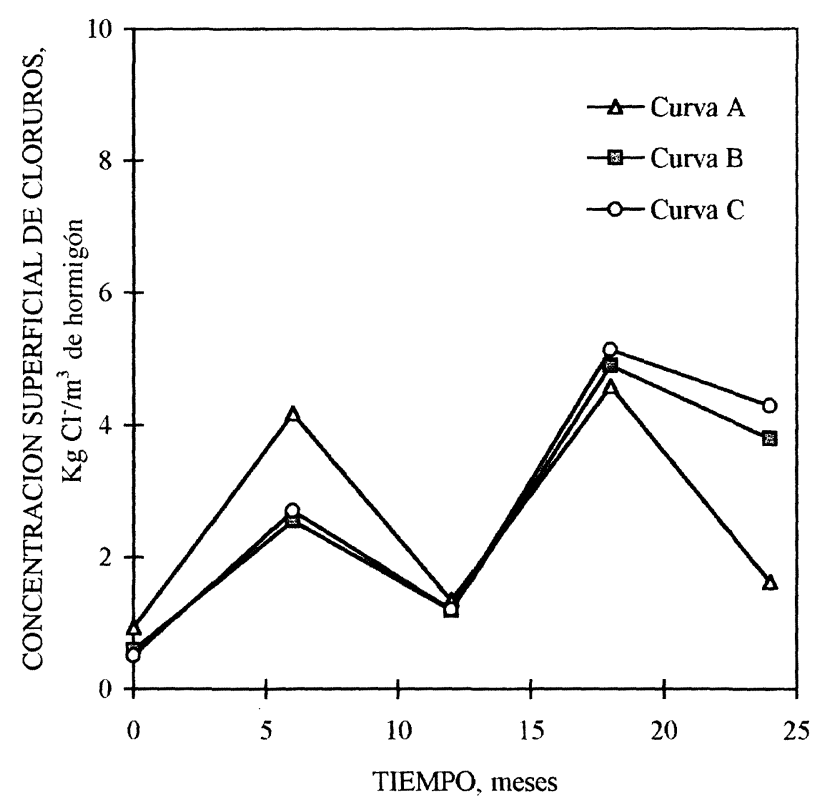

FIG. 3.- Concentración superficial de los iones cloruro en un cilindro de hormigón (datos experimentales) en función del tiempo de exposición. Curva A: relación a/c: 0,46, 7 d.c. Curva B: relación a/c: 0,70, 7 d.c. Curva C: relación a/c: 0,76, 1 d.c.

FIG. 3.- Surface concentration of chloride ions on a concrete cylinder as a function of exposure time (experimental data). Curve A: w/c: 0.46, curing time: 7 days. Curve B: $w / c: 0.70$, curing time:

7 days. Curve $C w / c$ 0.76, curing time: 1 day. $t^{\prime}=t-\phi$ para $\phi=0$ cuando $0<t<12 \mathrm{y}$

$\phi=12$ cuando $0<t<24$

donde $C_{\mathrm{s}}(t)$ es la concentración superficial (en kg $\mathrm{Cl}^{-} / \mathrm{m}^{3}$ de hormigón), $C_{\text {mín. la concentración míni- }}$ ma encontrada en el perfil de la gráfica, al igual que $C_{\text {máx. }}$ es la máxima, $t$ ' y $\phi$ son parámetros que consideran una frecuencia anual del fenómeno climático. En este caso, la frecuencia predominante en la región de Progreso, en Yucatán, es el tiempo en meses. Cada ecuación es la representación de la curva, ajustada a partir de los datos experimentales en un período de 2 años para las probetas de hormigón simple.

Para tomar en cuenta la variación del clima en la zona objeto del estudio, se propone una variabilidad sinusoidal para el valor de la concentración superficial de los iones cloruro en el hormigón durante el período de 2 años estudiado, considerándose una variación cíclica cada 6 meses, de acuerdo al patrón climático prevaleciente en la zona, caracterizado por una temporada de lluvias y otra seca. El inicio del seguimiento del proceso fue durante el mes de julio de 1993 que corresponde al principio de la estación de lluvias, la cual tiene una marcada influencia sobre la concentración superficial de los iones cloruro, tanto por el arrastre que sufren los cloruros presentes en la atmósfera hacia la superficie de las probetas como por el efecto de lavado. El siguiente período semestral tuvo lugar en el invierno, temporada seca, durante la cual la precipitación decae de manera importante, disminuyendo por consiguiente el arrastre de los cloruros ambientales. En cuanto a los otros dos semestres, el comportamiento es cualitativamente semejante, variando lógicamente los valores de las concentraciones.

\subsection{Modelo de difusión de cloruros}

Con el fin de considerar la variación de la concentración de cloruros superficial como una función del tiempo, se adoptó una función periódica de forma sinusoidal que describe dicha concentración tomando en cuenta la influencia de la concentración de los cloruros en el ambiente. Los resultados encontrados por el método propuesto se muestran en la figura 5. Comparando la figura 4 (perfil experimental) y la 5 (perfil simulado) se puede constatar que existe una buena concordancia cualitativa. Es interesante resaltar la importancia de los fenómenos climáticos sobre el fenómeno de difusión de los cloruros en el hormigón simple. La consideración del efecto del lavado y depósito superficial que tienen los períodos climáticos, permite explicar la naturaleza de los perfiles encontrados experimentalmente, mientras que la condición de frontera 


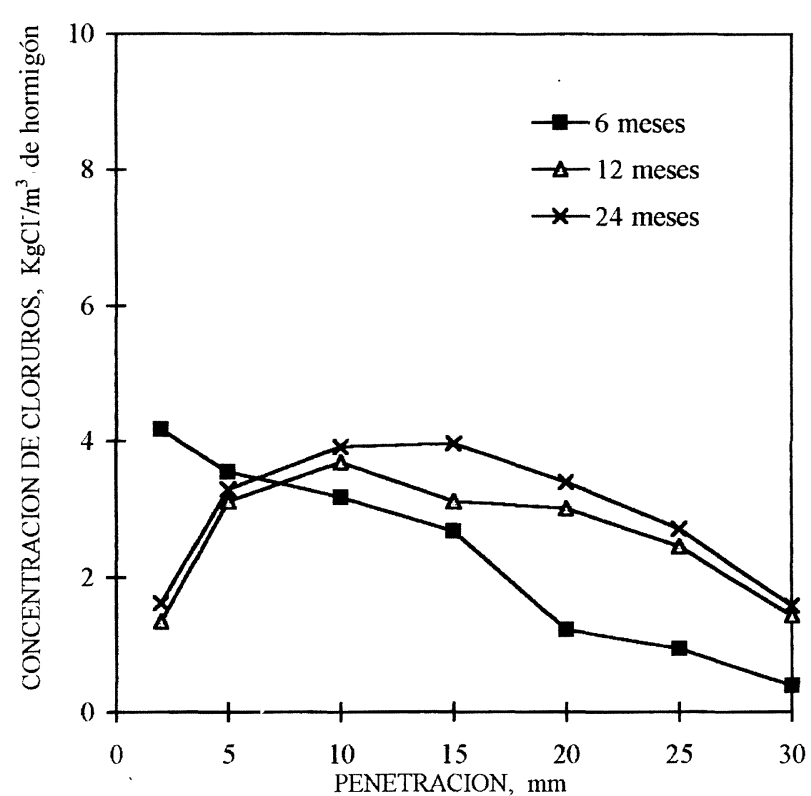

FIG. 4.- Perfil de concentración (datos experimentales) de los iones cloruro en una probeta cilíndrica de hormigón para diferentes tiempos de exposición. Relación a/c: 0,46, 7 d.c.

FIG. 4.- Concentration profile of chloride ions on a concrete cylinder at different exposure times (experimental data). $w / c: 0.46$, curing time: 7 days.

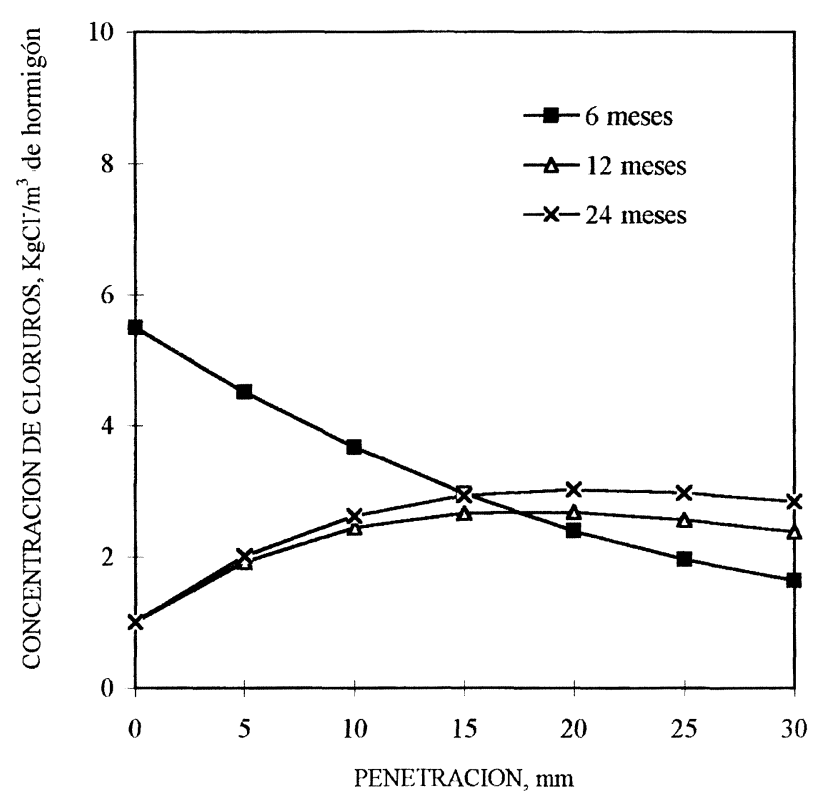

Fig. 5.- Perfil de concentración (datos simulados) de los iones cloruro en una probeta cilíndrica de hormigón para diferentes tiempos de exposición. Relación a/c: 0,46, 7 d.c.

FIG. 5.- Concentration profile (simulated) of chloride ions on a concrete cylinder at different exposure times. $w / c: 0.46$, curing time: 7 days. propuesta tiene una respuesta favorable para el modelo propuesto.

\section{CONCLUSIONES}

Se propone un modelo para el proceso de difusión de los iones cloruro en el hormigón basado en los siguientes parámetros: los valores de los coeficientes de difusión de los cloruros dependientes del tiempo $(t)$, una variable inherente al sistema como lo es el tiempo de curado (d.c.) y la concentración de cloruros en la superficie que varía con el tiempo $(t)$. Las soluciones numéricas de la ecuación diferencial gobernante del proceso han resultado una herramienta adecuada para la predicción del tiempo de exposición que tarda una estructura de hormigón en llegar a la concentración crítica, y así comenzar la corrosión del acero de refuerzo, para una forma geométrica cilíndrica, bajo las condiciones marinas prevalecientes en la Península de Yucatán (Méxiço).

\section{Agradecimientos}

Los autores agradecen al CINVESTAV, CONACyT (a través de los proyectos 211085-52186PA y FO5II9110), DGAPA y a la Facultad de Química de la UNAM, su apoyo económico en varias etapas de esta investigación.

\section{REFERENCIAS}

(1) Castro, P., Maldonado, L. y De Coss, R. Corros. Sci., 35 (5-8), 1993: 1.557-1.562.

(2) BerKe, N.S. y Hicks, M.C. Predicting chloride profiles in concrete. NACE Corrosion'94. Houston (Texas, EE.UU.), 1994.

(3) SAGuÉs, A.A. y KRANC, S.C. Proceedings of the Conference on Corrosion of Reinforcement Concrete Construction. Ed. Society for Chemical Industry. Londres, 1996.

(4) LIN, S.H. Corrosion (NACE), 46 (12), 1990: 964-967.

(5) Lin, S.H. Chem. Eng. Sci., 46 (4), 1991: 1.189-1.192.

(6) Saetta, A.V., Scotta, R.V. y Viatialiani, R.V. ACI Mater. J., 90 (5), 1993: 441-451.

(7) Sagués, A.A., Castro, P., Maldonado, L. Moreno, E. y Genesca, J. Proceedings of the First Mexican Symp. on Corrosion. Eds. L. Maldonado y M. Pech. Ed. Facultad Química. UNAM. México D.F. 1995: 43-52. 